\title{
A SOLUTION TO A SET OF FUNDAMENTAL EQUATIONS IN MARKOV CHAINS ${ }^{1}$
}

\author{
C. DERMAN
}

Let a Markov chain $\left\{X_{k}\right\}$ with stationary transition probabilities have the matrix of transition probabilities

$$
P=\left\{p_{i j}\right\} \quad(i, j=0,1,2, \cdots) .
$$

Then we have as usual

$$
\sum_{j=0}^{\infty} p_{i j}^{(n)}=1, \quad p_{i j}^{(n)} \geqq 0 \quad \text { for all } n, i \text { and } \jmath
$$

where $p_{i j}^{(n)}$ are elements of the matrix $P^{n}$. It is known (see [1, p. 325]) that when the states of the chain are aperiodic and neither transient nor null ${ }^{2}$ there exists a unique sequence $\left\{u_{k}\right\}$ of positive elements such that $\sum_{k=0}^{\infty} u_{k}=1$ and which satisfy the equations

$$
u_{k}=\sum_{i=0}^{\infty} u_{i} p_{i k} \quad \text { for } k=0,1, \cdots .
$$

If the chain is stationary, $u_{k}$ is the probability that at any given time the chain will be in state $k$. When the states are null no such set of probabilities exist since the $u_{k}$ 's would all be zero. However, while no probability can be ascribed to the chain being in a given state at a given time, the question arises as to whether some states are visited more frequently than others and whether relative frequencies considered in some sense have properties analogous to the set $\left\{u_{k}\right\}$. Doeblin [3] considered ratios of the type given in (2). He proved the existence of a finite positive limit for all types of chains.

We shall prove the following

THEOREM. If all the states of an irreducible chain are recurrent, then there exists one and only one set of positive numbers $\left\{v_{k}\right\}, v_{0}=1$ which satisfy the equations (1) and

$$
v_{k}=\lim _{n \rightarrow \infty} \frac{\sum_{r=0}^{n} p_{k k}^{(r)}}{\sum_{r=0}^{n} p_{00}^{(r)}} .
$$

Received by the editors March 23, 1953 and, in revised form, August 13, 1953.

${ }^{1}$ Work sponsored by the Office of Scientific Research of the Air Force.

${ }^{2}$ For definitions of all terms see [1]. 
Proof. ${ }^{3}$ Let ${ }_{k} p_{i j}^{n}=\operatorname{Pr}\left\{X_{n}=j, X_{m} \neq k\right.$ for $\left.1 \leqq m<n \mid X_{0}=i\right\}, n$ $=1,2, \cdots$. Chung [2] has shown that $\sum_{n=1}^{\infty} i p_{i j}^{n}={ }_{i} p_{i j}^{*}$ is positive and finite for all states $i$ and $j$. To prove that (2) satisfies (1) we note first that

$$
\begin{aligned}
\sum_{j=0}^{\infty}{ }_{0} p_{0 j}^{*} p_{j k} & ={ }_{0} p_{00}^{*} p_{0 k}+\sum_{j=1}^{\infty}{ }_{0} p_{0 j}^{*} p_{j k} \\
& =p_{0 k}+\sum_{n=1}^{\infty} \sum_{j=1}^{\infty}{ }_{0} p_{0 j}^{(n)} p_{j k}=p_{0 k}+\sum_{n=1}^{\infty}{ }_{0} p_{0 k}^{(n+1)} \\
& =0 p_{0 k}^{(1)}+\sum_{n=2}^{\infty}{ }_{0} p_{0 k}^{(n)}={ }_{0}^{*} p_{0 k}^{*} \quad \text { for } k=0,1, \cdots
\end{aligned}
$$

In the second equality we made use of the fact that ${ }_{0} p_{00}^{*}=1$. This is true since the states are recurrent. In [2] Chung has shown in the case where $i$ and $k$ are recurrent states that

$$
\lim _{n \rightarrow \infty} \frac{\sum_{r=0}^{n} p_{k k}^{(r)}}{\sum_{r=0}^{n} p_{i i}^{(r)}}={ }_{i} p_{i k}^{*} .
$$

Hence for $i=0$ we have shown that $v_{k}$ defined in (2) are positive solutions to (1).

To prove them unique let us suppose there exists a set $\left\{a_{k}\right\}$, $a_{0}=1$, of positive numbers satisfying (1). We define

$$
q_{i j}^{(n)}=\frac{a_{j}}{a_{i}} p_{j i}^{(n)} .
$$

Then, clearly, we have

$$
\sum_{j=0}^{\infty} q_{i j}^{(n)}=1 ; \quad q_{i j}^{(n)} \geqq 0 ; \quad q_{i j}^{(n+1)}=\sum_{k=0}^{\infty} q_{i k} q_{k j}^{(n)} .
$$

Thus the matrix $Q=\left\{q_{i j}\right\}(i, j=0,1,2, \cdots)$ is a matrix of transition probabilities. Now we can write

$$
\frac{\sum_{r=1}^{n} q_{i 0}^{(r)}}{\sum_{r=0}^{n} q_{00}^{(r)}}=\frac{1}{a_{i}} \frac{\sum_{r=1}^{n} p_{0 i}^{(r)}}{\sum_{r=0}^{n} p_{00}^{(r)}} .
$$

${ }^{3}$ The existence part of the proof given here was suggested by K. L. Chung. It replaces the original and more complicated proof. 
It is easily seen that $Q$ is the matrix of a chain whose states are recurrent. It follows from the work of Doeblin [3] or of Chung [2] that the left side of (6) tends to unity as $n \rightarrow \infty$. Hence we have that $a_{i}$ must be unique. This proves the theorem.

If we define recurrent states to be equally likely if all $v_{k}=1$ we have the following

Corollary 1. All recurrent states are equally likely if and only if $\sum_{i=0}^{\infty} p_{i j}=1$ for all $j$ (i.e., $P$ is doubly stochastic).

The above theorem together with the theorem given in $[1$, p. 325] implies

COROLlary 2. The states of an irreducible recurrent chain are null if and only if $\sum_{k=0}^{\infty} v_{k}=\infty$.

We remark that the notion of an inverse probability (see [1, p. 341 ]) can be extended by definition (4) to the case where all states are recurrent null. It is of interest to note that the solution to (1) need not be bounded. Examples showing this can easily be constructed. It is also of interest to note that the relation

$$
\lim _{n \rightarrow \infty} \frac{\sum_{r=1}^{n} p_{i j}^{(r)}}{\sum_{r=0}^{n} p_{i i}^{(r)}}={ }_{i} p_{i j}^{*}
$$

for all $i$ and $j$ proved by Chung [2] follows from (3) and (6) and the fact that the left side of (6) tends to unity as $n \rightarrow \infty$. However, Chung's proof is more general in that he does not assume the states to be recurrent.

I am indebted to Dr. T. E. Harris for calling my attention to this problem.

\section{REFERENCES}

1. W. Feller, An introduction to probability theory and its applications, Wiley, 1950.

2. K. L. Chung, Contributions to the theory of Markov chains, Journal of Research, National Bureau of Standards vol. 50 (1953).

3. W. Doeblin, Sur deux problemes de M. Kolmogoroff concernant les chaines denombrables, Bull. Soc. Math. France vol. 66 (1939).

Columbia University 